\title{
Enhanced recovery after surgery (ERAS) protocol for gastrectomy: A tailored program developed at a gastric cancer unit
}

\author{
Jacopo Desiderio ${ }^{1,2^{*}}$, Stefano Trastulli ${ }^{1}$, Antonio Di Cintio $^{1}$, Rita Commissari ${ }^{3}$, Andrea Colasanti ${ }^{3}$, Marialaura
} Scarcella ${ }^{3}$, Anna Mariniello ${ }^{4}$, Ilenia Grandone ${ }^{5}$, Sergio Bracarda ${ }^{6}$, Amilcare Parisi ${ }^{1}$

${ }^{1}$ Department of Digestive Surgery, St. Mary's Hospital, Terni, Italy.

${ }^{2}$ Department of Surgical Sciences, La Sapienza University of Rome - PhD program in advanced surgical technologies, Rome, Italy.

${ }^{3}$ Department of Anesthesiology and Intensive Care Medicine, St. Mary's Hospital, Terni, Italy.

${ }^{4}$ Department of Gastroenterology end Endoscopy, St. Mary's Hospital, Terni, Italy.

${ }^{5}$ Department of Diabetology, Dietology and Clinical Nutrition, St. Mary's Hospital, Terni, Italy.

${ }^{6}$ Medical Oncology Unit, St. Mary’s Hospital, Terni, Italy.

\section{To Cite}

Desiderio J, Trastulli S, Di Cintio A, Commissari R, Colasanti A, Scarcella M, Mariniello A, Grandone I, Bracarda S, Parisi A. Enhanced Recovery After Surgery (ERAS) Protocol for Gastrectomy: A Tailored Program Developed at a Gastric Cancer Unit. J Gastric Surg 2020; 2(1): $12-$ 17

\section{Pubblication history}

Received: February 2, 2020

Revised: February 26, 2020

Accepted: February 28, 2020

Article in press: February 29, 2020

Published online: March 15, 2020

\section{*Correspondence to}

Dr. Jacopo Desiderio

Department of Digestive Surgery,

St Mary's Hospital, Terni 05100, Italy

j.desiderio@aospterni.it

Telephone: +393425595828

\begin{abstract}
Background:

Planning for and managing patients who follow multidisciplinary paths allow institutions to provide better care administration; greater collaboration among medical staff, patients, and their relatives; better patients education; reduced possible complications related to surgery and hospital stay; and increased patient adherence to the proposed treatments due to better information. The ERAS Society's guidelines align in this direction, and many institutions are now looking to apply the suggestions contained in its items. This effort is especially important in surgical oncology. In this work, we report the experience of our center in developing tailored guidelines for patients undergoing gastrectomy based on evidence from the literature and adapted to address the availability of personnel and equipment in our institute.
\end{abstract}

Methods:

A permanent institutional working group was established at St. Mary's Hospital Evidence-based comprehensive research was conducted to find optimal perioperative care management for patients undergoing gastrectomy.

Evidence and recommendations were thoroughly evaluated and considered together with the items from the ERAS Society's guidelines.

Results:

A complete patient pathway has been established from the first outpatient visit to discharge.

All ERAS items were considered and adapted to our hospital's care environment Education, nutrition, anesthesiologist care, surgical approach, and ward organization are the main points of strength highlighted in the present work.

Conclusion:

This proposed institutional evidence-based protocol show comprehensive management for patients with gastric cancer eligible for enhanced surgical pathways.

Key words:

ERAS, Enhanced Recovery After Surgery, Gastrectomy, Gastric Cancer. 


\section{Background:}

Gastric cancer is the second leading cause of cancerrelated death globally, and surgery is the most important treatment of this disease. Even so, gastric cancer surgery remains a high-risk procedure that is associated with clinically significant postoperative stress, complications, and relevant sequelae. The morbidity and mortality of radical gastrectomy are $9.1-46.0 \%$ and $0-13 \%$, respectively.

In this context, ERAS programs have been proposed with which to improve postoperative physiological functionality and facilitate patient recovery. ERAS protocols have many elements, including preoperative patient education, preoperative loading of carbohydrates, nutrition from the first postoperative days, early mobilization of patients, and antithrombotic prophylaxis.

Briefly, we summarize the evidence relating to the points of greatest interest:

\section{Nasal-Gastric Tube, Abdominal Drainage, Mobilization}

No advantage is reported in the literature from the routine use of the nasogastric tube[1].

Some studies have shown that the nasogastric tube is not able to reduce the risk of anastomotic leakage, the number of lung complications, or mortality and that it significantly reduces the patient's postoperative comfort[2-4]. Furthermore, Yang's meta-analysis[5] indicated that postoperative maintenance of the tube prolongs the postoperative ileum and time to first flatus. Yamada[6] reported that complications that could be caused by a shortening of the postoperative fasting period, such as pneumonia ab-ingestis or anastomotic leakage, did not increase in a group of patients undergoing ERAS. In addition, the absence of abdominal drainage is an additional factor that improves patient comfort, stimulates and facilitates walking.

The evidence does not show any benefit to using abdominal drainage in numerous surgical procedures[ 7 , 8]. However, little evidence is available regarding gastric surgery. In particular, the use of drainages after total gastrectomy is still widely debated in the context of the development of ERAS programs.

An important item in the ERAS protocol is early mobilization[9], which is facilitated by absence of the tube and drainage as well as by early removal of the urinary catheter. Smart[10] has shown that failure of early patient mobilization is significantly associated with an extension of the postoperative stay.

Several studies[6, 11-13] have shown that application of these points of the ERAS program can significantly accelerate recovery of postoperative intestinal function compared with conventional management.

\section{Nutrition}

Consideration of functional outcomes such as first flatus or resumption of peristalsis can be at risk of bias. For this reason, it is appropriate to analyze in more detail variables related to recovery of oral intake.

ERAS protocols require that the patient not be subjected to long periods of fasting. Early nutrition has been shown to reduce postoperative catabolism, accelerate the return of intestinal function, and reduce the risk of complications[14, 15]. Furthermore, Lewis et al. [16] confirmed in their meta-analysis that keeping patients on an empty stomach brings no benefit. Several studies have shown that early oral nutrition not only is feasible in gastric surgery but also brings significant benefits[11, 17]; however, this point remains controversial.

Although early resumption of feeding has been shown to accelerate recovery of the patient after several surgical procedures, use of such an approach after gastrectomy has historically been viewed with distrust born out of a concern, not well demonstrated in the literature, that early oral intake could cause anastomotic leakage or intestinal obstruction.

Over the past few years, several studies have confirmed that early feeding after gastrectomy is safe and that it is associated with an improvement in functional recovery and a reduction in hospital stay[6, 18]. In particular, a randomized controlled trial reported data on safety in the resumption of oral feeding from the second postoperative day after gastrectomy[19].

Studies by Makuuchi[20] and Pedziwiatr[21], which contrasted use of an ERAS protocol and conventional management after gastrectomy, confirmed that resumption of oral nutrition is safe from the second postoperative day and that it is correlated with a reduction in postoperative administration of fluids intravenously as well as with early discharge[22].

Sugisawa[20] evaluated anastomotic leakage rate and pneumonia ab-ingestis to evaluate the real risk attributable to early nutrition. In this study, incidence of anastomotic leakage was $0.8 \%$ in the ERAS group-a figure not only lower than that of its historical comparison cohort $(1.7 \%)$ but also in line with or lower than data from previous studies reporting conventional perioperative management $(0.8-1.9 \%)$. Hence the author concluded that early oral nutrition does not adversely affect the anastomotic site. Similar results were obtained by Yamada[6, 23], who showed a similar incidence in incidence of leaks $(1.1 \%)$.

\section{Hospital Stay}

The effects of adopting an ERAS program on postoperative hospital stay depend not only on clinical factors but also on the health systems and sociocultural substrate of patients. For example, Yamada[6] reported that even though ERAS patients had a quicker functional recovery than those in the conventional group, length of stay did not significantly differ between the two groups. The authors attributed this result first to the Japanese Diagnosis Procedure Combination-based Payment System (DPC), which allows patients to extend their hospitalization at a reduced cost.

Among others, Sugisawa[20] reported that the median of postoperative hospital stay was significantly reduced in the ERAS group (8 days) compared with its historical cohort (10 days; $p=0.001)$. Similar results were obtained by Wang[11]. With regard to postoperative complications and the need for reoperation, all studies confirmed the safety of the ERAS approach and the absence of any statistically significant difference with the control groups[6,20].

In conclusion, it has been widely demonstrated that adoption of management based on ERAS principles in 
a reference center for gastric cancer can improve the patient's functional recovery and quality of life while allowing early discharge[24].

We show, in the present article, the ERAS Protocol approved at our gastric cancer unit.

\section{ERAS PROTOCOL}

\section{Eligibility of patients:}

Each patient must meet all the inclusion criteria and none of the exclusion criteria:

\section{Inclusion Criteria}

- Histological diagnosis of gastric cancer

- Preoperative staging performed by EGD and/or endoscopic ultrasound and CT, in accordance with international guidelines

- $\quad$ Early gastric cancer (EGC)

- Advanced gastric cancer (AGC)

- Patients treated with curative intent, in accordance with international guidelines

\section{Exclusion Criteria}

- Distant metastasis: peritoneal carcinosis, liver metastases, remote lymph node metastases, Krukenberg tumors, involvement of other organs

- Patients at high operative risk, as defined by the American Society of Anesthesiologists (ASA), score $\geq 4$

- History of previous abdominal surgery for gastric cancer

- Synchronous malignant tumor in other organs

- Palliative surgery

\section{Preoperative outpatient/home management:}

\section{Preoperative Counseling and Education}

The meeting with the patient must take place well in advance of the planned intervention and/or hospitalization in a dedicated environment (ERAS outpatient clinic) stocked with easily accessible and readily understandable information material, allowing for an interview between the patient and the multidisciplinary team (surgeon, anesthesiologist, nurse, dietician). The aim is to promote compliance with the protocol by sharing the objectives with the patient and motivating him or her to adhere to the path outlined. To this end, family members participate in the preoperative interview and assist the patient both during the hospitalization and once they return home. Counseling should take place sufficiently in advance of the scheduled admission date. It is highly recommended that the meeting take place in a multidisciplinary manner, with simultaneous participation of all professionals involved. Doing so allows all subjects to share health education and information data that the patient must receive, while avoiding repetition and finalizing the interview in an optimal way.

The anesthesiologist and surgeon should inform the patient of the relevant procedures and obtain informed consent. It is advisable that verbal information be integrated with delivery of informative material (brochures, brochures, videos, etc.).

\section{Assessment of Respiratory}

If the patient has a positive history of severe respiratory disease (COPD, asthma, sleep apnea syndrome), a clinical-instrumental evaluation of respiratory function is indicated, aimed at identifying subjects who could benefit from pre- and/or postoperative respiratory physiotherapy.

Nutritional and Behavioral Management in the Preoperative Period

- Assessment of nutritional status and dietary prescriptions. A preoperative nutritional risk assessment should be performed, preferably using the Malnutrition Universal Screening Tool (MUST https://www.bapen.org.uk/screeningand-must/must-calculator ) [25, 26]. Preoperative administration of immunonutrition is indicated for at least 5 days in all patients, and at least 7 days in malnourished patients, before surgery. The dietitian's evaluation is indicated in patients with a MUST score $\geq 2$.

- The patient should be asked to abstain from smoking and intake of all alcoholic beverages.

- In the days preceding the intervention (5 days), the patient should follow a special diet, as outlined during the outpatient visit.

- The patient is hospitalized the afternoon before surgery and from the start of the hospitalization can ingest only rusk, clear liquids, and dinners tailored by the dietetic and nutrition service.

- The patient may not consume food during the 6-8 $\mathrm{h}$ before surgery but might be able to consume clear liquids (clear fluids: water, tea, coffee, sports drinks, meat or vegetable broth, fruit juices without grape/apple/blueberry pulp, popsicles without pulp or pieces of fruit) up to 2-4 h before surgery.

- The patient must also be instructed in how to take the immunonutrient mixtures per OS. The protocol provides for the intake of $750 \mathrm{~mL} /$ day of product, starting 5 days before surgery (7 days in the malnourished patient).

- Administer a maltodextrin-based drink free of lipids, lactose, fiber, and gluten in the recommended dose of $800 \mathrm{~mL}$ the evening before the intervention and then, if the intervention occurs in the afternoon, another in a dose of $400 \mathrm{~mL} \mathrm{2-4} \mathrm{h}$ before the intervention. The drink should be taken fresh and not at room temperature.

\section{Intestinal Preparation}

No preparation of principle.

\section{Antithrombotic Prophylaxis \\ According to guidelines.}

\section{Antibiotic prophylaxis}

Administration of cefazoline $2 \mathrm{~g}$ IV $30 \mathrm{~min}$ before induction. 


\section{Anesthesiological Protocol}

Premedication

- Low doses of Midazolam $\sim 0.05 \mathrm{mg} / \mathrm{kg}$.

Type of anesthesia:

- general balanced with vapors: sevoflorane or desflurane associated with continuous infusion of short-acting opiates such as remifentanyl

or

- totally intravenous anesthesia (TIVA TCI) with propofol and remifentanyl in continuous infusion so as to associate anesthesiological depth control with BIS (bispectral index) sensor

- Use of fast-metabolizing curaries such as cisatracurium or those that guarantee a total reversal of neuromuscular blockade by sugammadex, such as rocuronium

- Continuous monitoring with skin temperature sensor

- $\quad$ Patient skin heating systems

Intraoperative fluid therapy optimization

- Heating of fluids infused to the patient

- EW1000 Edwards less invasive hemodynamic monitoring based on pulse contour method with headphone sensor or intra-arterial catheter for beatby-beat evaluation of cardiac output (CI) and stroke volume (SV)

- Optimization of intraoperative fluid therapy according to SV, based on the NICE protocol, to avoid edema of the intestinal mucosa and consequent slowing of motility due to overloading or underloading ischemias of the intestinal loops[27].

Intraoperative Pain and Postoperative Nausea and Vomiting (PONV) Control

- Transversus abdominis plane block (TAP block): Ropivacaine $0.2 \%(8-10 \mathrm{ml} / \mathrm{h})$ infused for $48-72 \mathrm{~h}$ through a multihole catheter.

- Use of Paracetamol and fans, infiltration of surgical wounds with long-acting local anesthetics such as levobupivacaine or ropivacaine for pain control

- Intraoperative prevention of postoperative nausea and vomiting according to Apfel score[28]

- Removal of the SNG if present before the end of the intervention

- In the event of open interventions, infiltration of the surgical wound with long-acting local anesthetics such as levobupivacaine or ropivacaine and placement of continuous-release catheters of local anesthetic at the suprafascial level.

\section{Surgical Technique}

- Surgical access: 2D/3D/4K laparoscopic, roboticassisted via the Da Vinci platform Yes. Laparotomic access is considered when the minimally invasive approach is not practicable.

- Drains: Not positioned in the distal gastrectomy. In the case of total gastrectomy, 1 drainage is positioned near the esophagus-jejunal anastomosis.

\section{Immediate Postoperative Monitoring}

- Transfer of the patient to recovery room

- Recovery of cognitive skills and evaluation according to Ramsay score

- After laparoscopic/robotic intervention continuous monitoring of $\mathrm{CO} 2$ in spontaneous breathing for 1 $\mathrm{h}$

- Pain assessment with analogue-visual VAS scale at 5,30 , and $60 \mathrm{~min}$

- Temperature control (time $0,3 \mathrm{~h}, 6 \mathrm{~h}$ )

\section{Postoperative Nausea and Vomiting (PONV)}

- The goal in ERAS is not to suspend liquid intake and oral feeding. Optimal control of symptoms (nausea and vomiting) with multimodal drug therapy (e.g., cortisone, ondansetron) should be guaranteed.

- In subjects who are at high risk of PONV (assessed on the basis of Apfel score), anti-emetic therapy should be prescribed, in principle[28].

\section{Prophylaxis of Postoperative Pain}

- Infiltration of surgical wounds with local anesthetic

- Administration of $1 \mathrm{~g}$ Paracetamol IV $20 \mathrm{~min}$ before the end of the intervention, repeated $4 \mathrm{~h}$ and $8 \mathrm{~h}$ apart

- $\quad$ Targin $20 \mathrm{mg}$ cpr for OS or ketorolac $30 \mathrm{mg}$ IV as needed

\section{Nutritional Management}

Specific nutritional protocol attached, but general principles include the following:

1. Preventing and/or managing malnutrition by default through nutritional risk assessment and gradual introduction of energy and nutrients until complete coverage needs are met.

2. Adaptation of diet to the new anotomic-functional capacities of the residual gastrointestinal tract and prevention or modulation of the different symptoms that can arise in the early postoperative period (sense of early satiety, nausea, vomiting, reflux, and dumping syndrome) through the following:

- $\quad$ splitting the diet into small and frequent meals (at least 6 meals/day)

- $\quad$ fluid intake between meals, reduced intake of foods and drinks rich in simple sugars due to their high osmotic power

- behavioral recommendations for meal management: eat slowly in small bites, chew well and sit upright for at least 30 to 60 minutes after the meal

\section{Resumption of Thromboembolic Prophylaxis}

Enoxaparin sodium starting from the 2 nd postoperative day and in accordance with the guidelines.

Use of Antibiotics

Avoid if not necessary. 
Infusion Therapy

Suspend when oral intake of fluids meets patient's water needs.

\section{Gastrografin Swallow}

1st postoperative day.

\section{Urinary Catheter Removal}

1st postoperative day.

\section{Start Mobilization}

- Encourage patient to mobilize as early as $2 \mathrm{~h}$ after returning to the ward.

- 1 1st day: patient must stay out of bed for at least $8 \mathrm{~h}$ and walk at least $600 \mathrm{~m}$.

- 2nd day: normal activity, not less than that prescribed for the 1st day.

- It is recommended that adequate rooms and armchairs be used to help the patient stay out of bed. It is useful for the patient to keep a diary in which to record time spent out of bed and, providing appropriate references, the precise distance walked.

\section{Respiratory Rehabilitation Using Incentive Spirometer 1st postoperative day.}

\section{Drainage Removal}

After execution of gastrografin swallow (1st postoperative day).

\section{Discharge criteria:}

- Ability to mobilize and independently practice personal hygiene care

- Free diet according to nutritional indications

- Adequate pain control with oral analgesics and VAS score $\leq 4$

- No clinical or laboratory evidence of postoperative complications or unresolved medical problems

- Patient consent

\section{Acknowledgements}

None.

\section{Contributors}

JD ST were involved in conception of the study. JD, ST, ADC, RC, AC, LS, AM, IG, SB, AP were involved in designing the study, analyzing the literature, references searching and in drafting the rationale. JD, ST, ADC, RC, AC, MS, AM, IG, SB, AP were involved in description of the study methods.

\section{Funding}

None.

\section{Competing interests}

No benefits in any form have been received or will be received from a commercial party related directly or indirectly to the subject of this article.

\section{Availability of data and materials}

The datasets used and/or analyzed during the current study are available

from the corresponding author on reasonable request.

\section{Appendix A. Supplementary data}

Supplementary data associated with this article can be found, in the on-line version at https://www. journalofgastricsurgery.com/index.php/JGS/article/ view/21

\section{Ethics approval}

Not applicable

\section{Provenance and peer review}

Not commissioned; externally peer reviewed.

\section{Open access}

This is an Open Access article distributed in accordance with the Creative Commons Attribution NonCommercial (CC BY-NC 4.0) license, which permits others to distribute, remix, adapt, build upon this work noncommercially, and license their derivative works on different terms, provided the original work is properly cited and the use is non-commercial. See: http:// creativecommons.org/licenses/by-nc/4.0/

\section{References}

[1] Abdikarim I, Cao XY, Li SZ, Zhao YQ, Taupyk Y, Wang Q. Enhanced recovery after surgery with laparoscopic radical gastrectomy for stomach carcinomas. World journal of gastroenterology. 2015;21:1333944.

[2] Suehiro T, Matsumata T, Shikada Y, Sugimachi K. Accelerated rehabilitation with early postoperative oral feeding following gastrectomy. Hepato-gastroenterology. 2004;51:1852-5.

[3] Carrere N, Seulin P, Julio CH, Bloom E, Gouzi JL, Pradere B. Is nasogastric or nasojejunal decompression necessary after gastrectomy? A prospective randomized trial. World journal of surgery. 2007;31:1227.

[4] Yoo CH, Son BH, Han WK, Pae WK. Nasogastric decompression is not necessary in operations for gastric cancer: prospective randomised trial. The European journal of surgery = Acta chirurgica. 2002;168:37983.

[5] Yang Z, Zheng Q, Wang Z. Meta-analysis of the need for nasogastric or nasojejunal decompression after gastrectomy for gastric cancer. The British journal of surgery. 2008;95:809-16.

[6] Yamada T, Hayashi T, Cho $\mathrm{H}$, Yoshikawa T, Taniguchi $\mathrm{H}$, Fukushima $\mathrm{R}$, et al. Usefulness of enhanced recovery after surgery protocol as compared with conventional perioperative care in gastric surgery. Gastric cancer : official journal of the International Gastric Cancer Association and the Japanese Gastric Cancer Association. 2012:15:34-41.

[7] Petrowsky H, Demartines N, Rousson V, Clavien PA. Evidencebased value of prophylactic drainage in gastrointestinal surgery: a systematic review and meta-analyses. Annals of surgery. 2004;240:107484; discussion 84-5.

[8] Kawai M, Tani M, Terasawa H, Ina S, Hirono S, Nishioka R, et al. Early removal of prophylactic drains reduces the risk of intraabdominal infections in patients with pancreatic head resection: prospective study for 104 consecutive patients. Annals of surgery. 2006;244:1-7.

[9] Vlug MS, Wind J, Hollmann MW, Ubbink DT, Cense HA, Engel $\mathrm{AF}$, et al. Laparoscopy in combination with fast track multimodal management is the best perioperative strategy in patients undergoing colonic surgery: a randomized clinical trial (LAFA-study). Annals of surgery. 2011;254:868-75.

[10] Smart NJ, White P, Allison AS, Ockrim JB, Kennedy RH, Francis NK. Deviation and failure of enhanced recovery after surgery following laparoscopic colorectal surgery: early prediction model. Colorectal disease : the official journal of the Association of Coloproctology of Great Britain and Ireland. 2012;14:e727-34.

[11] Wang D, Kong Y, Zhong B, Zhou X, Zhou Y. Fast-track surgery improves postoperative recovery in patients with gastric cancer: a randomized comparison with conventional postoperative care. Journal of gastrointestinal surgery : official journal of the Society for Surgery of 
the Alimentary Tract. 2010;14:620-7.

[12] Teeuwen PH, Bleichrodt RP, Strik C, Groenewoud JJ, Brinkert

W, van Laarhoven CJ, et al. Enhanced recovery after surgery (ERAS) versus conventional postoperative care in colorectal surgery. Journal of gastrointestinal surgery : official journal of the Society for Surgery of the Alimentary Tract. 2010;14:88-95.

[13] Desiderio J, Stewart CL, Sun V, Melstrom L, Warner S, Lee B, et al Enhanced Recovery after Surgery for Gastric Cancer Patients Improves Clinical Outcomes at a US Cancer Center. Journal of gastric cancer. 2018;18:230-41.

[14] Lewis SJ, Egger M, Sylvester PA, Thomas S. Early enteral feeding versus "nil by mouth" after gastrointestinal surgery: systematic review and meta-analysis of controlled trials. Bmj. 2001;323:773-6.

[15] Han-Geurts IJ, Hop WC, Kok NF, Lim A, Brouwer KJ, Jeekel J. Randomized clinical trial of the impact of early enteral feeding on postoperative ileus and recovery. The British journal of surgery. 2007;94:555-61.

[16] Lewis SJ, Andersen HK, Thomas S. Early enteral nutrition within $24 \mathrm{~h}$ of intestinal surgery versus later commencement of feeding: a systematic review and meta-analysis. Journal of gastrointestinal surgery : official journal of the Society for Surgery of the Alimentary Tract. 2009;13:569-75.

[17] Jo DH, Jeong O, Sun JW, Jeong MR, Ryu SY, Park YK. Feasibility study of early oral intake after gastrectomy for gastric carcinoma. Journal of gastric cancer. 2011;11:101-8.

[18] Scatizzi M, Kroning KC, Boddi V, De Prizio M, Feroci F. Fast-track surgery after laparoscopic colorectal surgery: is it feasible in a general surgery unit? Surgery. 2010;147:219-26.

[19] Hur H, Kim SG, Shim JH, Song KY, Kim W, Park CH, et al. Effect of early oral feeding after gastric cancer surgery: a result of randomized clinical trial. Surgery. 2011;149:561-8.

[20] Makuuchi R, Sugisawa N, Kaji S, Hikage M, Tokunaga M, Tanizawa $\mathrm{Y}$, et al. Enhanced recovery after surgery for gastric cancer and an assessment of preoperative carbohydrate loading. European journal of surgical oncology : the journal of the European Society of Surgical Oncology and the British Association of Surgical Oncology. 2016.

[21] Pedziwiatr M, Matlok M, Kisialeuski M, Migaczewski M, Major P, Winiarski M, et al. Short hospital stays after laparoscopic gastric surgery under an Enhanced Recovery After Surgery (ERAS) pathway: experience at a single center. European surgery : ACA : Acta chirurgica Austriaca. 2014;46:128-32.

[22] Terashima M. The earlier the better? Gastric cancer : official journal of the International Gastric Cancer Association and the Japanese Gastric Cancer Association. 2014;17:197-9.

[23] Yamada T, Hayashi T, Aoyama T, Shirai J, Fujikawa H, Cho H, et al. Feasibility of enhanced recovery after surgery in gastric surgery: a retrospective study. BMC surgery. 2014;14:41.

[24] Desiderio J, Trastulli S, D'Andrea V, Parisi A. Enhanced recovery after surgery for gastric cancer (ERAS-GC): optimizing patient outcome. J Translational Gastroenterology Hepatology. 2019;5.

[25] Elia M. The 'MUST' report. Nutritional screening for adults: a multidisciplinary responsibility. Development and use of the 'Malnutrition Universal Screening Tool' (MUST) for adults. 2003.

[26] Stratton RJ, Hackston A, Longmore D, Dixon R, Price S, Stroud M, et al. Malnutrition in hospital outpatients and inpatients: prevalence, concurrent validity and ease of use of the 'malnutrition universal screening tool' ('MUST') for adults. Br J Nutr. 2004;92:799-808.

[27] Rollins KE, Lobo DN. Intraoperative Goal-directed Fluid Therapy in Elective Major Abdominal Surgery: A Meta-analysis of Randomized Controlled Trials. Annals of surgery. 2016;263:465-76.

[28] Pierre S, Whelan R. Nausea and vomiting after surgery. Continuing Education in Anaesthesia Critical Care \& Pain. 2012;13:28-32. 